\title{
A cadaveric precision and accuracy analysis of augmented reality-mediated percutaneous pedicle implant insertion
}

\author{
Presented at the 2020 AANS/CNS Joint Section on Disorders of the Spine and Peripheral Nerves \\ Camilo A. Molina, MD, ${ }^{1}$ Frank M. Phillips, MD, ${ }^{3}$ Matthew W. Colman, MD, ${ }^{3}$ Wilson Z. Ray, MD, ${ }^{1}$ \\ Majid Khan, MD, ${ }^{2}$ Emanuele Orru', MD, ${ }^{4}$ Kornelis Poelstra, MD, PhD, ${ }^{5}$ and Larry Khoo, MD $^{6}$
}

\begin{abstract}
'Department of Neurosurgery, Washington University School of Medicine in St. Louis, Missouri; ${ }^{2}$ Department of Radiology, Johns Hopkins University School of Medicine, Baltimore, Maryland; ' ${ }^{3}$ Department of Orthopedic Surgery, Rush University School of Medicine, Chicago, Illinois; " Department of Neurointerventional Radiology, Lahey Clinic Tufts University School of Medicine, Boston, Massachusetts; ${ }^{5}$ The Robotic Spine Institute of Silicon Valley at OrthoNorCal, Los Gatos, California; and ${ }^{6}$ The Spine Clinic of Los Angeles, California
\end{abstract}

OBJECTIVE Augmented reality-mediated spine surgery (ARMSS) is a minimally invasive novel technology that has the potential to increase the efficiency, accuracy, and safety of conventional percutaneous pedicle screw insertion methods. Visual 3D spinal anatomical and 2D navigation images are directly projected onto the operator's retina and superimposed over the surgical field, eliminating field of vision and attention shift to a remote display. The objective of this cadaveric study was to assess the accuracy and precision of percutaneous ARMSS pedicle implant insertion.

METHODS Instrumentation was placed in 5 cadaveric torsos via ARMSS with the xvision augmented reality headmounted display (AR-HMD) platform at levels ranging from T5 to $\mathrm{S} 1$ for a total of 113 total implants (93 pedicle screws and 20 Jamshidi needles). Postprocedural CT scans were graded by two independent neuroradiologists using the Gertzbein-Robbins scale (grades A-E) for clinical accuracy. Technical precision was calculated using superimposition analysis employing the Medical Image Interaction Toolkit to yield angular trajectory $\left(^{\circ}\right)$ and linear screw tip (mm) deviation from the virtual pedicle screw position compared with the actual pedicle screw position on postprocedural CT imaging.

RESULTS The overall implant insertion clinical accuracy achieved was $99.1 \%$. Lumbosacral and thoracic clinical accuracies were $100 \%$ and $98.2 \%$, respectively. Specifically, among all implants inserted, 112 were noted to be GertzbeinRobbins grade A or B (99.12\%), with only 1 medial Gertzbein-Robbins grade C breach (> 2-mm pedicle breach) in a thoracic pedicle at T9. Precision analysis of the inserted pedicle screws yielded a mean screw tip linear deviation of 1.98 $\mathrm{mm}(99 \% \mathrm{Cl} 1.74-2.22 \mathrm{~mm})$ and a mean angular error of $1.29^{\circ}\left(99 \% \mathrm{Cl} 1.11^{\circ}-1.46^{\circ}\right)$ from the projected trajectory. These data compare favorably with data from existing navigation platforms and regulatory precision requirements mandating that linear and angular deviation be less than $3 \mathrm{~mm}(p<0.01)$ and $3^{\circ}(p<0.01)$, respectively.

CONCLUSIONS Percutaneous ARMSS pedicle implant insertion is a technically feasible, accurate, and highly precise method.

https://thejns.org/doi/abs/10.3171/2020.6.SPINE20370

KEYWORDS computer-navigated surgery; neuronavigation; cadaveric trial; robotic spine surgery; augmented reality-assisted surgery; mixed reality-assisted surgery; minimally invasive spine surgery; percutaneous pedicle screw insertion; surgical technique

$\mathrm{P}$ ERCUTANEOUs pedicle screw instrumentation has become a mainstay in minimally invasive thoracolumbar spinal fixation for a broad range of spinal pathologies. ${ }^{1-4}$ Conventionally, it is performed using biplanar fluoroscopy; ${ }^{1,5}$ however, advances in computer navigation and, most recently, robotics, are aimed at minimizing screw misplacement, decreasing radiation exposure, and improving procedural efficiency. ${ }^{6,7}$ Although modern manual and robot-assisted computer neuronavigation methods have demonstrated excellent screw insertion accuracy rates, there are technical disadvantages to both methods. ${ }^{1,4}$ A commonly cited challenge is line-of-sight (LOS) inter-

ABBREVIATIONS AR-HMD = augmented reality head-mounted display; ARMSS = augmented reality-mediated spine surgery; LOS = line of sight; MITK = Medical Image Interaction Toolkit; UBL = upper-bound limit.

SUBMITTED March 16, 2020. ACCEPTED June 1, 2020.

INCLUDE WHEN CITING Published online October 30, 2020; DOI: 10.3171/2020.6.SPINE20370. 
ruption in which live computer navigation is interrupted by a field obstruction prohibiting the visualization of tracking markers by the remote tracking camera, resulting in the loss of navigation until the obstruction is cleared. Manual computer-navigated systems have a delayed learning curve associated with the required skill of directing instruments based on imaging visualized on a remote screen, the ability to replicate inline maneuvers while exchanging instruments, ${ }^{8}$ and, most importantly, attention shift, ${ }^{9}$ where operators must shift their attention away from the patient to the remote screen for guidance. Attention shift has been documented to negatively affect both cognitive and motor tasks and is a source of error in percutaneous pedicle screw misplacement. ${ }^{10}$ Current iterations of robot-assisted computer-navigated spine surgery essentially reflect the use of standard computer navigation with a robotic arm providing an alignment guide for the placement of screws. Adoption pitfalls include errors with preoperative planning, shifts in the entry point and/or trajectory when paraspinal soft tissue is excessive, instruments sliding off angled bone surfaces such as hypertrophied facet joints, and attention shift. ${ }^{10-13}$

The current study evaluated a head-mounted display augmented reality-mediated spine surgery (ARMSS) system that uses wireless optical tracking cameras directly embedded inside the headset (Fig. 1A), minimizing LOS interruption. The system projects $3 \mathrm{D}$ and $2 \mathrm{D}$ reconstructed models along with tracking information directly onto the operator's retina, using a transparent near-eye-display headset to visualize both the surgical area (Fig. 1C-E) and navigation data in the same field of view, thereby eliminating the attention-shift disadvantage inherent to the remote navigation displays of both manual and robotic computer navigation systems. Molina et al. ${ }^{10}$ previously published the first proof-of-concept cadaveric study, demonstrating excellent clinical accuracy employing an augmented reality head-mounted display (AR-HMD; xvision, Augmedics Ltd.) for open thoracolumbar pedicle instrumentation. Here, to the best of our knowledge, we present the first cadaveric trial employing an AR-HMD for the percutaneous placement of thoracic and lumbosacral pedicle screws.

\section{Methods \\ Cadaveric Trial Design}

Five fresh-frozen cadaveric torsos with no previous history of spine surgery were procured. The torsos were positioned prone on a CT-integrated table (AIRO, Brainlab; Fig. 2A). A small incision was made above L1-2 to enable insertion of a reference clamp to the spinous processes of L1-2 (Fig. 2B). Registration clamps provide a navigation range of approximately 8 vertebral segments cranially and caudally. Hence, clamp attachment at L1-2 permitted registration and navigation over the T5-S1 vertebral segments. A registration marker was then attached to the clamp (Z-marker, Augmedics; Fig. 2C).

The DICOM data from a CT scan (AIRO; Fig. 2A) was then transferred to the xvision console and processed for an automatic 3D segmentation. The registration marker was then exchanged for a reflective navigation marker (XVS Marker, Augmedics; Fig. 2D). The reflective naviga- tion marker was attached to the reference clamp and then flipped opposite to the working side (i.e., if working on the right side, the reflective patient marker was flipped to be most prominent on the left side to free any potential obstacle to instrumentation on the right side, and vice versa when working on the left side).

Four attending spine surgeons and one spine surgery fellow participated in pedicle screw insertion using the AR-HMD (Fig. 1). The participants were trained on the system using phantoms. Each cadaver was registered for possible instrumentation from the T5 to S1 vertebrae, yielding 140 possible individual pedicles, of which 113 were randomly chosen for percutaneous instrumentation: 93 pedicle screws and 20 Jamshidi needles. The AR-HMD provided a direct retinal projection displaying a 3D reconstruction (Fig. 1C) of the specimen spines as well as axial (Fig. 1D) and sagittal (Fig. 1E) instrument trajectories to guide pedicle instrumentation.

\section{Instrument Insertion Method}

The surgeons were asked to make a $1.5-\mathrm{cm}$ skin incision based only on the retinal image projected by the 3D model. The 3D model was then used to percutaneously traverse the soft tissue with a Jamshidi needle directly down to the bony pedicle screw entry point. This technique allowed for 3D visualization of the spine and surgical landmarks. It also allowed a simultaneous in-projection view of standard sagittal and axial 2D CT images, as with traditional computer navigation. The surgeons subsequently referenced the axial and sagittal trajectory projections to finalize craniocaudal and mediolateral virtual trajectories for optimal pedicle cannulation (Fig. 1D) and executed this using the Jamshidi needle. This virtual trajectory was then saved for display on both the 2D and 3D projections (Fig. 1E). The Jamshidi needle was then removed and exchanged for a pedicle screw using the saved virtual trajectory to guide the operator back into the cannulated Jamshidi needle track denoted as a red circle virtual track (Fig. $1 \mathrm{C})$. No K-wires were used. Twenty Jamshidi (11-gauge diameter and 6 inches in length) needles were not exchanged for pedicle screws in order to independently evaluate Jamshidi needle insertion precision and accuracy. All inserted thoracic pedicle screws (Premia Spine) were $5.5 \mathrm{~mm}$ in diameter and $40 \mathrm{~mm}$ in length. All inserted lumbar pedicle screws (Premia Spine) were $6.5 \mathrm{~mm}$ in diameter and $45-50 \mathrm{~mm}$ in length. To minimize the risk of inaccuracies due to spine movements during instrumentation, registration of the lumbosacral area was performed first, followed by instrumentation of that area and subsequent postprocedural scan. This process was then repeated for the thoracic instrumentation.

\section{Radiographic Technical Precision Analysis}

After completion of the instrumentation, the reflective marker was exchanged for the registration marker, and a postprocedural CT scan was obtained. Postprocedural registration was required to transform the coordinates of the postprocedural scan to the coordinate system of the preprocedural scan for precision analysis. Specifically, the postprocedural scan was loaded into the Medical 
Molina et al.
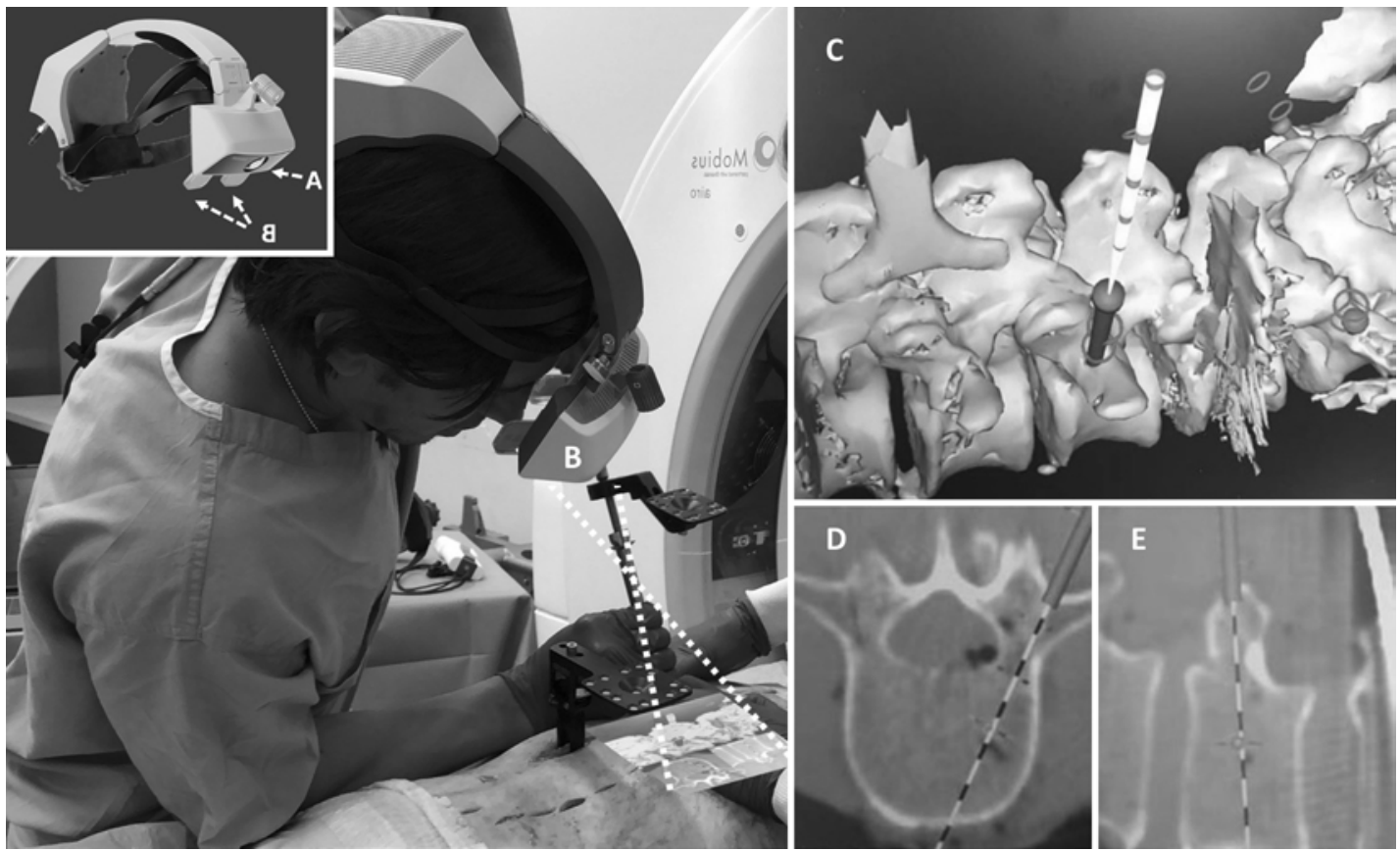

FIG. 1. A-C: AR-HMD employed with the head-mounted optical infrared tracking camera $(A)$ and transparent near-eye-display (B) providing the 3D reconstruction of the spine specimen (C). D and E: Axial (D) and sagittal (E) trajectories of the tracked instruments. The red circles in panel $\mathrm{C}$ represent the phantom pedicle cannulation track by Jamshidi needle and are saved to guide insertion of the pedicle screw. Figure is available in color online only.

Image Interaction Toolkit (MITK) software application. The 3D actual position of the implant tip and crown were determined visually in pixel coordinates. As depicted in Fig. 3, the actual implant tip and crown were captured in axial, coronal, and sagittal projections. The implant tip and crown points were manually marked and verified to match in all three projections. The 3D spatial calculation positioning of the implant tip (st:[x,y,z]) and crown
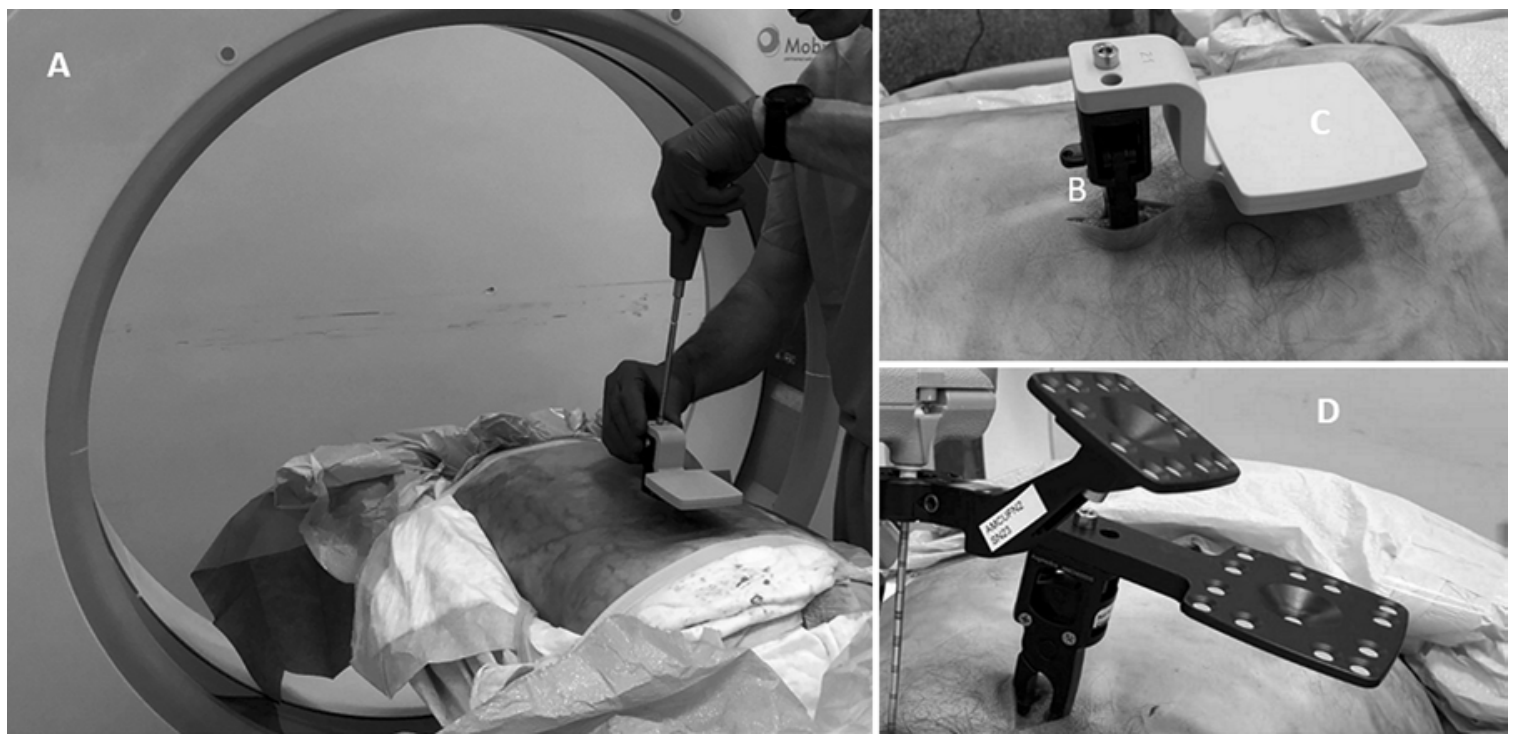

FIG. 2. A: Cadaveric torso in the prone position on the AIRO-integrated CT table. B: Spinous process reference clamp placement over the L1-2 spinous processes. C: Placement of the registration marker on the spinous process clamp. D: Exchange of registration marker for reflective navigation marker that can be flipped from left to right to minimize LOS interruption. Figure is available in color online only. 

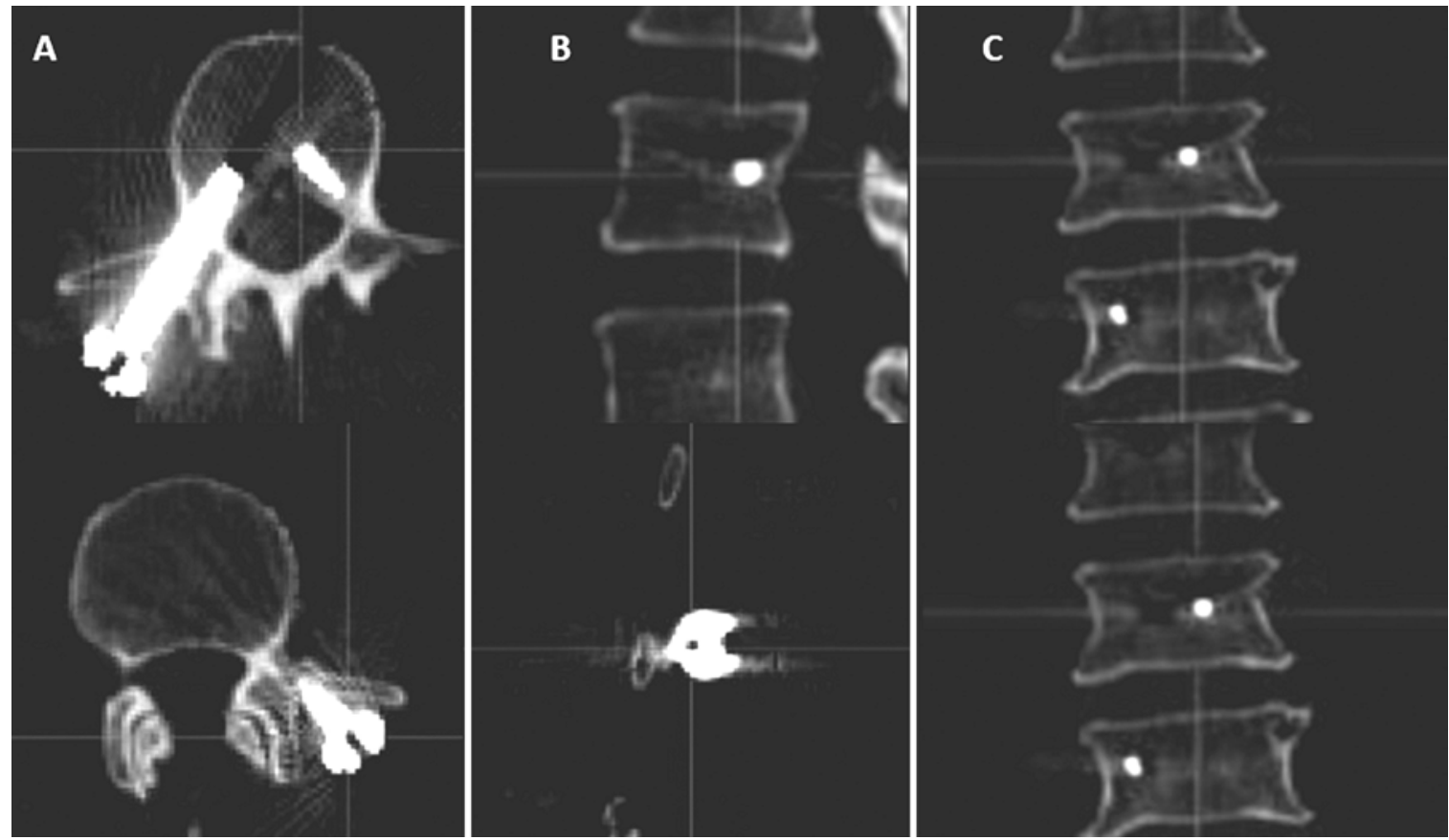

FIG. 3. A: Axial view of the 3D positional measurements $(x, y, z)$ for the actual screw tip and crown. B and C: Sagittal (B) and coronal (C) projections on the MITK application.

(sc:[x,y,z]) was performed automatically by the MITK software. These coordinates were transformed from the DICOM volume voxels to the specimen marker reference coordinate system in millimeters using the postprocedural scan registration transformation detailed in Appendix 1. Linear tip error $(\mathrm{mm})$ and angular trajectory error $\left(^{\circ}\right)$ were analyzed for all inserted pedicle screws and stratified according to screws inserted in the lumbosacral and thoracic vertebrae.

\section{Radiographic Accuracy Analysis}

Postprocedural CT scans of each cadaveric sample were obtained and submitted to two neuroradiologists for independent accuracy grading. Both lumbar and thoracic pedicle instrumentation placement accuracies were assessed using the Gertzbein-Robbins scale, ${ }^{14}$ where grade A and $\mathrm{B}(<2-\mathrm{mm}$ pedicle wall breach)-classified pedicle instrumentation are considered clinically accurately placed. Scoring disagreement between the two neuroradiologists was resolved by joint evaluation with an independent third party trained and practiced in spine surgery.

\section{Statistical Analysis}

Descriptive statistics were used to calculate the mean, standard deviation, maximal value, range, $99 \%$ confidence interval, and upper-bound limit (UBL) for the linear tip $(\mathrm{mm})$ and trajectory $\left({ }^{\circ}\right)$ deviations of virtual versus actual implant positions. Additionally, a one-sample t-test was used to compare the measured errors with predefined regulatory navigation precision criteria requiring $<3 \mathrm{~mm}$ and $<3^{\circ}$ of linear and trajectory deviation, respectively. All radiological and statistical analyses were performed by independent investigators.

\section{Results \\ Clinical Accuracy Analysis}

Accuracy analyses were performed using the Gertzbein-Robbins ${ }^{14}$ grading scheme, defining grade A or B as clinically acceptable. The total implant insertion accuracy was $99.1 \%$. Accuracies of all thoracic and lumbosacral implants were $98.2 \%$ and $100 \%$, respectively. Stratification according to implant type yielded a Jamshidi insertion accuracy of $100 \%$ and pedicle screw insertion accuracy of 98.9\%. Stratification of pedicle screw insertion accuracy by segment yielded a $100 \%$ lumbosacral insertion accuracy and $97.9 \%$ thoracic insertion accuracy. Specifically, only 1 of 93 pedicle screws was misplaced, when a $5.5-\mathrm{mm}-$ diameter screw placed within a 3.7-mm-bicortical-width pedicle resulted in a 3.9-mm medial canal breach (Fig. 4). No Jamshidi needles were misplaced. Total, segmental, and individual cadaver insertion accuracies are listed in Table 1 and Supplementary Table 1.

\section{Technical Precision Analysis}

Precision analyses were performed by calculating the linear $(\mathrm{mm})$ and angular $\left({ }^{\circ}\right)$ deviation of the real pedicle screw position compared with that of the virtual pedicle screw trajectories; $\mathrm{p}$ values represent a one-tailed t-test of the $99 \%$ CI UBL versus accepted computer navigation precision safety standards of $3 \mathrm{~mm}$ and $3^{\circ}$ of linear and angular deviation, respectively. ${ }^{15}$ The overall pedicle screw linear deviation per cadaver ranged from 1.63 to $2.20 \mathrm{~mm}$ (mean $1.98 \pm 0.90 \mathrm{~mm}$ [99\% CI UBL $2.22 \mathrm{~mm}$ ], p < 0.01). The thoracic pedicle screw linear deviation per cadaver ranged from 1.43 to $2.26 \mathrm{~mm}$ (mean $1.93 \pm 0.82 \mathrm{~mm}[99 \%$ CI UBL $2.24 \mathrm{~mm}$ ], $\mathrm{p}<0.01$ ). The lumbosacral pedicle screw linear deviation per cadaver ranged from 1.59 to 

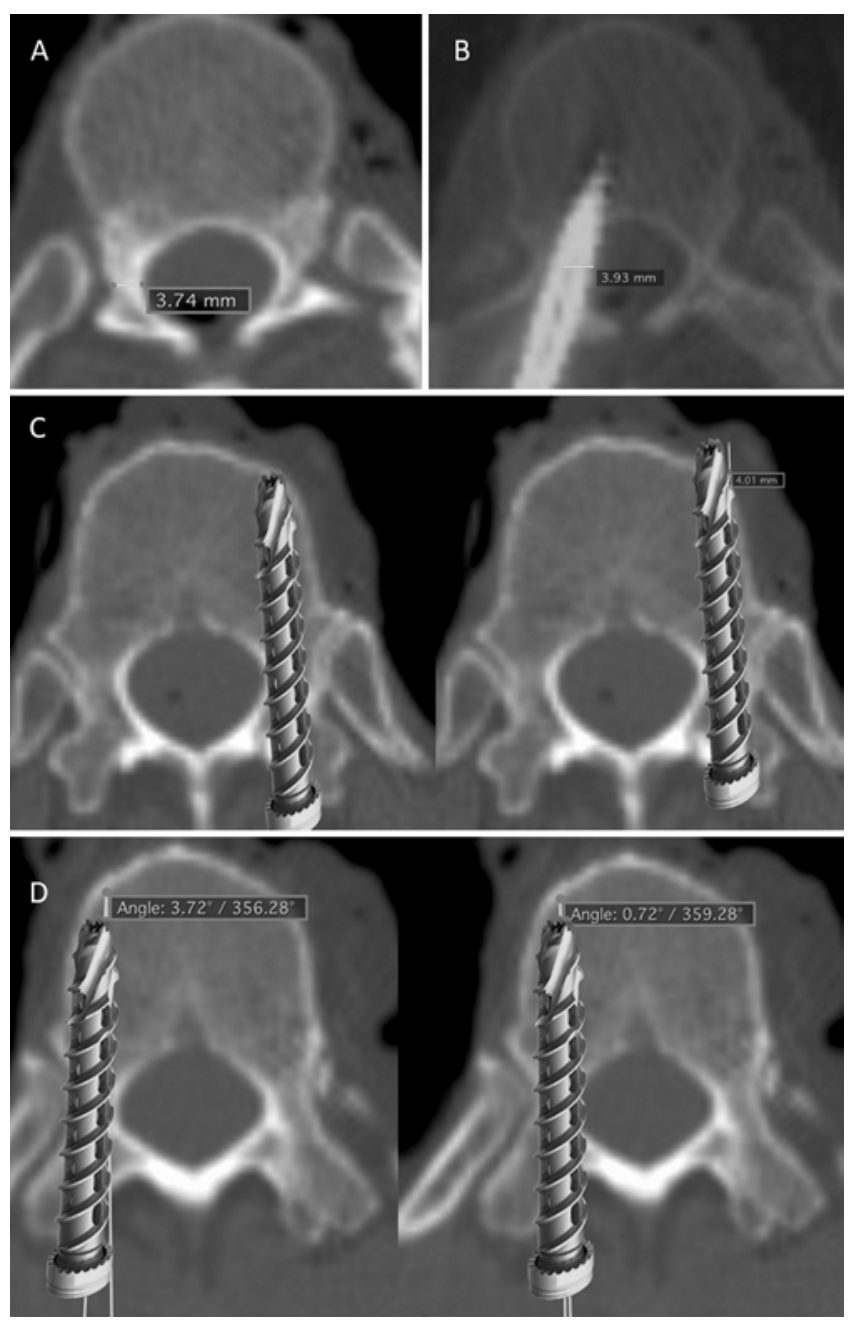

FIG. 4. A and B: Preoperative axial projections demonstrating a single 3.9-mm medial canal breach of a $3.7-\mathrm{mm}$ T9 pedicle by a $5.5-\mathrm{mm}$ pedicle screw. C: Model demonstration of anterior breach when attempting to maximize the screw length, but the navigation system has a 4-mm linear tip deviation. D: Demonstration of a medial breach due to the $3^{\circ}$ angular trajectory deviation. Figure is available in color online only.

$2.41 \mathrm{~mm}$ (mean $2.03 \pm 0.99 \mathrm{~mm}$ [99\% CI UBL $2.40 \mathrm{~mm}$ ], $\mathrm{p}<0.01)$. The linear deviation values per individual screw and associated statistical analysis are listed in Table 2 and Supplementary Table 2. The overall pedicle screw angular deviation per cadaver ranged from $1.13^{\circ}$ to $1.40^{\circ}$ (mean $1.29^{\circ} \pm 0.65^{\circ}\left[99 \% \mathrm{CI}\right.$ UBL $\left.\left.1.46^{\circ}\right], \mathrm{p}<0.01\right)$. The thoracic pedicle screw angular deviation per cadaver ranged from $0.91^{\circ}$ to $1.45^{\circ}$ (mean $1.17^{\circ} \pm 0.67^{\circ}\left[99 \%\right.$ CI UBL $1.42^{\circ}$ ], p $<0.01$ ). The lumbosacral pedicle screw angular deviation per cadaver ranged from $1.25^{\circ}$ to $1.81^{\circ}$ (mean $1.41^{\circ} \pm 0.61^{\circ}$ $\left[99 \%\right.$ CI UBL $\left.\left.1.64^{\circ}\right], \mathrm{p}<0.001\right)$. Angular deviation values per individual screw and associated statistical analysis are listed in Table 3 and Supplementary Table 3.

\section{Discussion}

Modern minimally invasive spine surgery has evolved to provide solutions for the surgical management of most spinal pathologies including, but not limited to, degen-
TABLE 1. Insertional accuracy for Jamshidi needle and percutaneous pedicle screw implants

\begin{tabular}{lccc}
\hline & \multicolumn{3}{c}{ Insertional Accuracy } \\
\cline { 2 - 4 } & Total Implant & Jamshidi Needle & Pedicle Screw \\
\hline Total & $99.1 \%(112 / 113)$ & $100 \%(20 / 20)$ & $98.9 \%(92 / 93)$ \\
\hline Thoracic & $98.2 \%(56 / 57)$ & $100 \%(10 / 10)$ & $97.9 \%(46 / 47)$ \\
\hline Lumbosacral & $100 \%(56 / 56)$ & $100 \%(10 / 10)$ & $100 \%(46 / 46)$ \\
\hline
\end{tabular}

erative, traumatic, oncological, and deformity spine disease. ${ }^{16-19}$ Percutaneous pedicle screw instrumentation is the most prevalent solution to achieve posterior spinal fixation in the setting of preexisting or iatrogenic mechanical instability during minimally invasive surgery procedures. ${ }^{1,4}$ Classically, percutaneous pedicle screw instrumentation is performed with biplanar fluoroscopy. This technique has reported insertion accuracy rates ranging from $83.9 \%$ to $100 \%$ and is associated with varying amounts of radiation exposure to the patient and staff, depending on the clinical team's workflow and experience. , $, 20,21^{\text {Manual computer }}$ navigation utilizing a remote infrared camera positioned outside the surgical field has improved accuracy rates ranging from $96.3 \%$ to $100 \%{ }^{2,5-7,21-28}$ In addition to improved accuracy, computer navigation has the advantage of performing K-wireless ${ }^{7}$ percutaneous screw insertion and minimizing radiation exposure to the clinical team and potentially the patient, depending on the registration method used. ${ }^{21}$ Despite improvements in accuracy, with manual computer navigation drawbacks have commonly been reported that may result in procedural inefficiency and error. As previously discussed, these include failure to replicate inline maneuvers during tool exchange (particularly with K-wireless techniques), loss of live navigation due to LOS interruption, and attention shift.,10,29,30 Attention shift in particular negatively impacts cognitive and motor tasks because it requires cyclical redirection of operator attention from the surgical field to a remote screen for guidance. . $^{910}$

The next evolution, robotic computer-navigated spine surgery, retains the advantages of manual computer navigation, but improves on them by eliminating the need to replicate inline maneuvers by employing a stereotactic end-effector working port. ${ }^{11-13,31-37}$ However, robotic computer navigation is still prone to attention-shift errors and procedural inefficiency associated with LOS obstruction. LOS interruption occurs when targeting is based on remote optical tracking cameras that must simultaneously

TABLE 2. Descriptive statistics for linear tip deviation and a one-sample t-test used to compare the measured errors versus predefined regulatory navigation precision criteria requiring $<3$ $\mathrm{mm}$ of linear deviation

\begin{tabular}{lccccc}
\hline & No. of Screws & Mean & SD & $99 \%$ UBL & $p$ Value \\
\hline Total & 93 & 1.98 & 0.90 & 2.22 & $<0.01$ \\
\hline Thoracic & 47 & 1.93 & 0.82 & 2.24 & $<0.01$ \\
\hline Lumbosacral & 46 & 2.03 & 0.99 & 2.40 & $<0.01$ \\
\hline
\end{tabular}


TABLE 3. Descriptive statistics for angular trajectory deviation and a one-sample t-test used to compare the measured errors versus predefined regulatory navigation precision criteria requiring $<3^{\circ}$ of angular deviation

\begin{tabular}{lccccc}
\hline & No. of Screws & Mean & SD & $99 \%$ UBL & $p$ Value \\
\hline Total & 93 & 1.29 & 0.65 & 1.46 & $<0.01$ \\
\hline Thoracic & 47 & 1.17 & 0.67 & 1.42 & $<0.01$ \\
\hline Lumbosacral & 46 & 1.41 & 0.61 & 1.64 & $<0.01$ \\
\hline
\end{tabular}

track the position of the reference registration frame, the tracked instrument, and the robot end-effector. ${ }^{29,36,37}$ Occlusion or exclusion of any of those key components from the remote tracking camera's field of view results in workflow interruption that introduces inefficiency and potential targeting errors. ${ }^{36,37}$ Additional potential pitfalls include errors with preoperative planning, shifts in the entry point and/or trajectory when traversed soft tissue is excessive, and instruments sliding on angled bony surfaces such as hypertrophied facet joints. ${ }^{10-13,38}$

AR-HMDs with headset-integrated tracking cameras (Fig. 2A) and translucent direct retinal near-eye displays (Fig. 2B) improve on currently available computer navigation by eliminating attention shift and minimizing LOS interruption. LOS interruption sources can be divided into extrinsic (i.e., outside the operator's working field) and intrinsic (i.e., inside the operator's working field) sources. Extrinsic obstructions (e.g., staff, assistants, instruments, drapes, trays) can block the remote tracking camera's visualization but are excluded in this platform by employing an AR-HMD-integrated tracking camera (Fig. 1A). Intrinsic LOS obstructions mainly refer to the operator positioning instruments in a manner that obstructs the tracking camera's visualization of the instrument fiducials, reference frame, or both. Intrinsic LOS obstruction can still occur in the present AR-HMD system. To reestablish navigation using the AR-HMD system, the operator can change the position of the tracked instrument, adjust the position of the AR-HMD-integrated camera via small adjustments in the position of his or her head, or both. The AR-HMD-integrated camera ultimately mitigates LOS obstructions by eliminating extrinsic LOS obstruction sources and allowing for rapid correction of intrinsic LOS interruptions.

This technique also provides for virtual 3D visualization of the spinal anatomy as well as standard 2D CT navigation images. We previously published an open cadaveric study with this platform and demonstrated excellent user experience and noninferior open pedicle screw insertion clinical accuracy in comparison with manual computer and robot navigation data reported in the literature..$^{10}$ The aim of the present study was to evaluate the clinical accuracy and technical precision of ARMSS percutaneously inserted pedicle screws using the present AR-HMD platform.

Most spine instrument navigation studies present clinical accuracy as Gertzbein-Robbins grade A- or B-positioned pedicle implants. ${ }^{35,39}$ The grade is interpreted as a system's ability to guide insertion of a pedicle implant with less than $2 \mathrm{~mm}$ of pedicle wall breach, independent of breach direction. ${ }^{14}$ The present AR-HMD system yielded a total implant clinical accuracy of $99.1 \%$ for all inserted Jamshidi needles and pedicle screws. Jamshidi needle insertion accuracy was $100 \%$ for both thoracic and lumbosacral segment stratification. The total percutaneous pedicle screw insertion accuracy was $98.9 \%$. When stratified according to thoracic or lumbosacral segments, percutaneous pedicle screw insertional accuracy was $97.9 \%$ and $100 \%$, respectively (Table 1). Of 93 screws, only one $5.5-\mathrm{mm}$ screw within a 3.6-mm-bicortical-width T9 pedicle had a 3.9-mm medial wall breach (Fig. 4). The present clinical accuracy rates compare favorably with literature reporting percutaneous pedicle screw insertion accuracy rates for manual computer-navigated methods (range 96\%$100 \%),{ }^{6,723-28,40}$ particularly for the lower reported thoracic insertion accuracies ranging from $75.6 \%$ to $94.9 \%$, where the anatomical threshold for error is smaller. ${ }^{6,25}$ The present results also compare favorably with robot-navigated percutaneous insertion, where reported clinical accuracy rates range widely from $89 \%$ to $100 \%$, depending on the timing of publication and robot platform used. . $^{12,13,31-37,41}$

Although positional clinical accuracy is important, it does not evaluate a navigation system's technical precision. Precision analysis evaluates the system's fidelity by determining the positional (linear and angular) deviation of the real implant compared with the virtual implant (i.e., how well the position of the real implant matches the position of the virtual navigated implant). Few studies have reported on technical precision. ${ }^{36,37,42-44}$ Precision data can be analyzed by studying deviation in 3 planes (i.e., 3D $[\mathrm{x}, \mathrm{y}, \mathrm{z}]$ ) or 2 planes (i.e., 2D [x,y]). The 3D and 2D analyzed data precision results cannot be compared directly, as 3D analysis incorporates an additional plane of variability and typically yields greater amplitude in both linear and angular deviation. Kleck et al ${ }^{42}$ were the first to report a method for calculating 3D linear and angular deviation for 157 pedicle screws percutaneously inserted via StealthStation computer navigation (Medtronic Inc.) and found 3D linear and angular deviations of $5.92 \mathrm{~mm}$ and $3.09^{\circ}$, respectively. Jiang et al. ${ }^{36}$ analyzed 8 percutaneously inserted screws using the ExcelsiusGPS robot (Globus Medical) in 2 patients and found a 2D linear deviation of $2.1 \mathrm{~mm}$ and angular deviation of $2.4^{\circ}$. Elmi-Terander et al. ${ }^{44}$ performed a cadaveric study inserting 66 Jamshidi needles using a novel remote screen AR hybrid system (XperCT, Philips Healthcare) and found a 2D linear deviation of 2.2 $\mathrm{mm}$ and angular deviation of $0.9^{\circ}$. Godzik et al.$^{37}$ reported both $2 \mathrm{D}$ and $3 \mathrm{D}$ precision analysis for 116 percutaneously inserted pedicle screws via the ExcelsiusGPS robot, with an overall insertion Gertzbein-Robbins clinical accuracy of $96.6 \%$. They found a mean linear deviation of $5.0 \mathrm{~mm}$ and $2.6 \mathrm{~mm}$ for $3 \mathrm{D}$ and $2 \mathrm{D}$ analyzed precision, respectively, highlighting the ample incongruity of $3 \mathrm{D}$ versus 2D plane precision analysis. The present AR-HMD's 3D precision analysis (Fig. 3) found an overall 3D linear deviation of $1.98 \pm 0.90 \mathrm{~mm}$ and angular deviation of $1.29^{\circ}$ $\pm 0.65^{\circ}$ (Tables 2 and 3). These findings compare favorably with the 3D precision deviation results reported by Kleck et al. ${ }^{42}$ (linear deviation: $5.92 \mathrm{~mm}$; angular deviation: $3.09^{\circ}$ ) and Godzik et al. ${ }^{37}$ (linear deviation: $5.0 \mathrm{~mm}$; angular deviation: $5 \cdot 6^{\circ}$ ). Additionally, both total and spine 
segment-stratified linear and angular deviation precision results were statistically significantly lower $(p<0.01)$ than the regulatory navigation standard of $<3 \mathrm{~mm}$ and $<3^{\circ}$ of deviation $^{15}$ (Tables 2 and 3).

Understanding precision deviation is critical for the safe use of navigation technologies. For example, surgeons must incorporate knowledge of and compensate for a navigation system's known linear deviation error when attempting to maximize screw insertion length to avoid breaching the anterior vertebral body cortex with risk of visceral or vascular injury (Fig. 4C). Similarly, precision in angular deviation is critical, particularly in small atrophic pedicles where small angular deviations can result in pedicle wall breaches (Fig. 4D). Correspondingly, Godzik et al. ${ }^{37}$ observed that all medially breached screws paradoxically had a plan that included less than $2 \mathrm{~mm}$ of the medial cortical wall, thereby leading their group to modify their robot trajectory planning to be greater than $2 \mathrm{~mm}$ from the cortical wall to avoid a medial canal breach. Hence, understanding and compensating for the systematic error of different navigation systems' precision allows the operator to use the technology in the safest method possible.

Limitations of this study include those inherent to cadaveric studies, comparison with historic controls, and potential sources of error during precision analysis. Cadaveric studies have decreased sources of external LOS obstruction; however, this is less relevant in this setting due to the absence of a remote tracking camera. Additionally, a notable difference between live clinical scenarios and cadaveric studies is the potential for motion due to patient respiration negatively affecting registrational/navigational accuracy. The absence of this source of motion may positively bias registrational and navigational precision and accuracy results in cadaveric studies. There is also the possibility of screw-tip measurement error with the MITK platform. The screws that we used were cannulated screws with a blunt rather than a sharp tip. Hence, the visually marked screw tip could fall between 2 pixels. As the scan resolution was $1 \mathrm{~mm}$, a single-pixel difference in marking could account for a $0.5-\mathrm{mm}$ difference in the defined position of the actual screw tip. In addition, mechanical inaccuracies might introduce error. If there is any motion between the interface of the screw and screwdriver, the toggle results in positional incongruency between the real and virtual implant.

User experience drawbacks when employing AR-mediated navigation for the placement of spine instrumentation include mechanical discomfort, visual discomfort, and visual obstruction. Although the AR-HMD platform is designed to be lightweight and only worn during hardware insertion, there is still a possibility for surgeon mechanical discomfort and fatigue while wearing the device. Users must adapt to having visual data directly projected to the retina and mixed with real visual objects. This creates an opportunity for visual discomfort that dissipates as users adapt to the projected data. Although the projected visual data are translucent, they may still obstruct visualization of key anatomical structures. Avoidance of this issue has a learning curve. It can be avoided by toggling on/off the projected images with a foot pedal or adjusting one's gaze to view the area of interest outside the retinal projection of the navigation data. We previously published a surgeon user-experience analysis and found these sources of discomfort to be minimal and not prohibitive to user adoption..$^{10}$

\section{Conclusions}

ARMSS via an AR-HMD is a clinically accurate and technically precise method for the insertion of percutaneous pedicle implants without many of the disadvantages implicit in traditional computer navigation and robot-assisted screw placement techniques. To our knowledge, we present the first cadaveric trial using an AR-HMD for percutaneous insertion of thoracic and lumbosacral pedicle instrumentation.

\section{References}

1. Proietti L, Scaramuzzo L, Schirò GR, et al. Posterior percutaneous reduction and fixation of thoraco-lumbar burst fractures. Orthop Traumatol Surg Res. 2014;100(5):455-460.

2. Yang BP, Wahl MM, Idler CS. Percutaneous lumbar pedicle screw placement aided by computer-assisted fluoroscopybased navigation: perioperative results of a prospective, comparative, multicenter study. Spine (Phila Pa 1976). 2012; 37(24):2055-2060.

3. Tinelli M, Matschke S, Adams M, et al. Correct positioning of pedicle screws with a percutaneous minimal invasive system in spine trauma. Orthop Traumatol Surg Res. 2014; 100(4):389-393.

4. Chapman TM, Blizzard DJ, Brown CR. CT accuracy of percutaneous versus open pedicle screw techniques: a series of 1609 screws. Eur Spine J. 2016;25(6):1781-1786.

5. Ohba T, Ebata S, Fujita K, et al. Percutaneous pedicle screw placements: accuracy and rates of cranial facet joint violation using conventional fluoroscopy compared with intraoperative three-dimensional computed tomography computer navigation. Eur Spine J. 2016;25(6):1775-1780.

6. Tajsic T, Patel K, Farmer R, et al. Spinal navigation for minimally invasive thoracic and lumbosacral spine fixation: implications for radiation exposure, operative time, and accuracy of pedicle screw placement. Eur Spine J. 2018;27(8): 1918-1924.

7. Spitz SM, Sandhu FA, Voyadzis JM. Percutaneous "K-wireless" pedicle screw fixation technique: an evaluation of the initial experience of 100 screws with assessment of accuracy, radiation exposure, and procedure time. J Neurosurg Spine. 2015;22(4):422-431.

8. Nottmeier EW. A review of image-guided spinal surgery. $J$ Neurosurg Sci. 2012;56(1):35-47.

9. Léger É, Drouin S, Collins DL, et al. Quantifying attention shifts in augmented reality image-guided neurosurgery. Healthc Technol Lett. 2017;4(5):188-192.

10. Molina CA, Theodore N, Ahmed AK, et al. Augmented reality-assisted pedicle screw insertion: a cadaveric proof-ofconcept study. J Neurosurg Spine. 2019;31(1):139-146.

11. Fiani B, Quadri SA, Ramakrishnan V, et al. Retrospective review on accuracy: a pilot study of robotically guided thoracolumbar/sacral pedicle screws versus fluoroscopy-guided and computerized tomography stealth-guided screws. Cureus. 2017;9(7):e1437.

12. Laudato PA, Pierzchala K, Schizas C. Pedicle screw insertion accuracy using $\mathrm{O}$-arm, robotic guidance or freehand technique: a comparative study. Spine (Phila Pa 1976). 2018; 43(6):E373-E378.

13. Molliqaj G, Schatlo B, Alaid A, et al. Accuracy of robotguided versus freehand fluoroscopy-assisted pedicle screw insertion in thoracolumbar spinal surgery. Neurosurg Focus. 2017;42(5):E14. 
14. Gertzbein SD, Robbins SE. Accuracy of pedicular screw placement in vivo. Spine (Phila Pa 1976). 1990;15(1):11-14.

15. Melkerson M. Stryker navigation system with SpineMap go software application, fluoroscopy trackers and fluoroscopy adapters, SpineMask tracker. Federal Drug Administration; 2019.

16. Eck JC, Hodges S, Humphreys SC. Minimally invasive lumbar spinal fusion. J Am Acad Orthop Surg. 2007;15(6): 321-329.

17. Foley KT, Holly LT, Schwender JD. Minimally invasive lumbar fusion. Spine (Phila Pa 1976). 2003;28(15 Suppl): S26-S35.

18. O'Toole JE, Eichholz KM, Fessler RG. Minimally invasive approaches to vertebral column and spinal cord tumors. $\mathrm{Neu}$ rosurg Clin N Am. 2006;17(4):491-506.

19. Holly LT, Schwender JD, Rouben DP, Foley KT. Minimally invasive transforaminal lumbar interbody fusion: indications, technique, and complications. Neurosurg Focus. 2006;20(3): E6.

20. Park DK, Thomas AO, St Clair S, Bawa M. Percutaneous lumbar and thoracic pedicle screws: a trauma experience. $J$ Spinal Disord Tech. 2014;27(3):154-161.

21. Zhang L, Zhou X, Cai X, et al. Reduction in radiation during percutaneous lumbar pedicle screw placement using a new device. Minim Invasive Ther Allied Technol. 2014;23(3): 173-178.

22. Innocenzi G, Bistazzoni S, D'Ercole M, et al. Does navigation improve pedicle screw placement accuracy? comparison between navigated and non-navigated percutaneous and open fixations. Acta Neurochir Suppl. 2017;124:289-295.

23. Siasios ID, Pollina J, Khan A, Dimopoulos VG. Percutaneous screw placement in the lumbar spine with a modified guidance technique based on 3D CT navigation system. J Spine Surg. 2017;3(4):657-665.

24. Fraser J, Gebhard H, Irie D, et al. Iso-C/3-dimensional neuronavigation versus conventional fluoroscopy for minimally invasive pedicle screw placement in lumbar fusion. Minim Invasive Neurosurg. 2010;53(4):184-190.

25. Kakarla UK, Little AS, Chang SW, et al. Placement of percutaneous thoracic pedicle screws using neuronavigation. World Neurosurg. 2010;74(6):606-610.

26. Kim TT, Drazin D, Shweikeh F, et al. Clinical and radiographic outcomes of minimally invasive percutaneous pedicle screw placement with intraoperative CT $(\mathrm{O}$-arm) image guidance navigation. Neurosurg Focus. 2014;36(3):E1.

27. Houten JK, Nasser R, Baxi N. Clinical assessment of percutaneous lumbar pedicle screw placement using the O-arm multidimensional surgical imaging system. Neurosurgery. 2012;70(4):990-995.

28. Bourgeois AC, Faulkner AR, Bradley YC, et al. Improved accuracy of minimally invasive transpedicular screw placement in the lumbar spine with 3-dimensional stereotactic image guidance: a comparative meta-analysis. J Spinal Disord Tech. 2015;28(9):324-329.

29. Rahmathulla G, Nottmeier EW, Pirris SM, et al. Intraoperative image-guided spinal navigation: technical pitfalls and their avoidance. Neurosurg Focus. 2014;36(3):E3.

30. Mummaneni PV, Rodts GE Jr. The mini-open transforaminal lumbar interbody fusion. Neurosurgery. 2005;57(4 Suppl): 256-261.

31. Gao S, Lv Z, Fang H. Robot-assisted and conventional freehand pedicle screw placement: a systematic review and meta-analysis of randomized controlled trials. Eur Spine J. 2018;27(4):921-930.

32. Marcus HJ, Cundy TP, Nandi D, et al. Robot-assisted and fluoroscopy-guided pedicle screw placement: a systematic review. Eur Spine J. 2014;23(2):291-297.

33. Liu H, Chen W, Wang Z, et al. Comparison of the accuracy between robot-assisted and conventional freehand pedicle screw placement: a systematic review and meta-analysis. Int J CARS. 2016;11(12):2273-2281.

34. Keric N, Doenitz C, Haj A, et al. Evaluation of robot-guided minimally invasive implantation of 2067 pedicle screws. Neurosurg Focus. 2017;42(5):E11.

35. van Dijk JD, van den Ende RP, Stramigioli S, et al. Clinical pedicle screw accuracy and deviation from planning in robotguided spine surgery: robot-guided pedicle screw accuracy. Spine (Phila Pa 1976). 2015;40(17):E986-E991.

36. Jiang B, Karim Ahmed A, Zygourakis CC, et al. Pedicle screw accuracy assessment in ExcelsiusGPS® robotic spine surgery: evaluation of deviation from pre-planned trajectory. Chin Neurosurg J. 2018;4(1):23.

37. Godzik J, Walker CT, Hartman C, et al. A quantitative assessment of the accuracy and reliability of robotically guided percutaneous pedicle screw placement: technique and application accuracy. Oper Neurosurg (Hagerstown). 2019;17(4): 389-395.

38. Nicholas K, Yeatts SD, Zhao W, et al. The impact of covariate adjustment at randomization and analysis for binary outcomes: understanding differences between superiority and noninferiority trials. Stat Med. 2015;34(11):1834-1840.

39. Mason A, Paulsen R, Babuska JM, et al. The accuracy of pedicle screw placement using intraoperative image guidance systems. J Neurosurg Spine. 2014;20(2):196-203.

40. Sadrameli SS, Jafrani R, Staub BN, et al. Minimally invasive, stereotactic, wireless, percutaneous pedicle screw placement in the lumbar spine: accuracy rates with 182 consecutive screws. Int J Spine Surg. 2018;12(6):650-658.

41. Schröder ML, Staartjes VE. Revisions for screw malposition and clinical outcomes after robot-guided lumbar fusion for spondylolisthesis. Neurosurg Focus. 2017;42(5):E12.

42. Kleck CJ, Cullilmore I, LaFleur M, et al. A new 3-dimensional method for measuring precision in surgical navigation and methods to optimize navigation accuracy. Eur Spine J. 2016;25(6):1764-1774.

43. Miller CA, Ledonio CG, Hunt MA, et al. Reliability of the planned pedicle screw trajectory versus the actual pedicle screw trajectory using intra-operative 3D CT and image guidance. Int J Spine Surg. 2016;10:38.

44. Elmi-Terander A, Nachabe R, Skulason H, et al. Feasibility and accuracy of thoracolumbar minimally invasive pedicle screw placement with augmented reality navigation technology. Spine (Phila Pa 1976). 2018;43(14):1018-1023.

\section{Disclosures}

All of the operators have financial relationships with Augmedics Ltd. as listed below. However, radiological and statistical analysis were completed by authors free of any financial relationship or bias related to Augmedics Ltd.

Dr. Molina: consultant for Augmedics and SurgeonMR. Dr. Phillips: direct stock ownership in Augmedics. Dr. Colman: direct stock ownership in Augmedics. Dr. Khan: consultant for Stryker Medical and MedWaves AveCure. Dr. Poelstra: clinical or research support for the study described from Augmedics. Dr. Khoo: direct stock ownership in Augmedics.

\section{Author Contributions}

Conception and design: Molina, Phillips. Acquisition of data: Molina, Phillips, Khan, Orru', Poelstra, Khoo. Analysis and interpretation of data: Molina, Khan, Orru'. Drafting the article: Molina. Critically revising the article: Molina, Phillips, Colman, Ray, Khan, Poelstra, Khoo. Reviewed submitted version of manuscript: all authors. Approved the final version of the manuscript on behalf of all authors: Molina. Administrative/technical/material support: Molina, Colman, Poelstra, Khoo. 


\section{Supplemental Information}

Online-Only Content

Supplemental material is available with the online version of the article.

Supplementary Tables and Appendix 1. https://thejns.org/doi/ suppl/10.3171/2020.6.SPINE20370.

\section{Previous Presentations}

Portions of this paper were presented at the 36th Annual Meeting of the AANS/CNS Section on Disorders of the Spine and Peripheral Nerves, Las Vegas, Nevada, March 8, 2020.

\section{Correspondence}

Camilo A. Molina: Washington University School of Medicine in St. Louis, MO.molina.a.camilo@gmail.com. 